Pathologe $2021 \cdot 42: 55-63$

https://doi.org/10.1007/s00292-020-00903-8

Angenommen: 17. Dezember 2020

Online publiziert: 18. Januar 2021

(c) Springer Medizin Verlag GmbH, ein Teil von

Springer Nature 2021

Schwerpunktherausgeber

S. Perner, Lübeck

F. Stellmacher, Borstel

\author{
Rosemarie Kruparr ${ }^{1,2} \cdot$ Christiane Kümpers $^{2} \cdot$ Alexander Haenel $^{3} \cdot$ Sven Perner ${ }^{1,2,4}$. \\ Florian Stellmacher ${ }^{1}$ \\ 'Pathologie, Forschungszentrum Borstel, Leibniz Lungenzentrum, Borstel, Deutschland \\ ${ }^{2}$ Institut für Pathologie, Universität zu Lübeck und Universitätsklinikum Schleswig-Holstein, Campus \\ Lübeck, Lübeck, Deutschland \\ ${ }^{3}$ Klinik für Radiologie und Nuklearmedizin, Universitätsklinikum Schleswig-Holstein, Campus Lübeck, \\ Lübeck, Deutschland \\ ${ }^{4}$ Airway Research Center North, Deutsches Zentrum für Lungenforschung, Großhansdorf, Deutschland
}

\title{
Kryptogen organisierende Pneumonie versus sekundäre organisierende Pneumonie
}

che damit eine Abgrenzung zur postinfektiösen OP darstellen wollten [8]. Sie beschrieben damals weiterhin die hohe Glukokortikoidsensitivität der OP und ihren Neigung zum Rezidiv bei zu frühzeitigen Absetzen der Medikation [5]. Insgesamt ist die Epidemiologie der OP schlecht dokumentiert. Eine isländische Studie spricht von einer Inzidenz von 1,10/100.000 Einwohner für die COP und $0,87 / 100.000$ für sekundäre Formen der OP [13]. Nichtsdestotrotz ist die OP eine häufig beobachtbare histologische Veränderung bei der pathologischen Aufarbeitung interstitieller Lungenerkrankungen oder sekundär im Rahmen anderer primärer Lungenerkrankungen. Klinisch zeigen COP-Patienten subakute Symptome wie über Wochen, meist über Monate andauerndes Fieber, Husten und/oder Dyspnoe. Das histologische Hauptcharakteristikum der OP sind Mesenchymknospen aus Granulationsgewebe, sogenannte Masson-Körper, welche in einem bronchiolozentrischen Verteilungsmuster die Lungenalveolen ausfüllen. Differenzialdiagnostisch sind die organisierende Form des DAD und die gewöhnliche interstitielle Pneumonie („usual interstitial pneumonia“, UIP) zu berücksichtigen. Auch hier sind Fibroblastenproliferate $\mathrm{zu}$ finden, welche aber intraseptal und nicht intraalveolar verordnet sind. Die COP ist glukokortikoidsensitiv und hat eine exzellente Prognose. Therapie und Prognose anderer Formen der OP richten sich nach der beteiligten Grunderkrankung.

\section{COP}

Die idiopathische oder kryptogene OP („cryptogenic organizing pneumonia“, COP) wurde 2002 durch eine Arbeitsgruppe der American Thoracic Society (ATS) und der European Respiratory Society (ERS) als eine Form der idiopathischen interstitiellen Pneumonien (IIP) oder auch interstitiellen Lungenerkrankungen („interstitial lung disease“, ILD) definiert [1]. Im Jahr 2013 erfolgte eine Überarbeitung der IIP-Einteilung durch die ATS/ERS. Die COP wird seitdem zu einer der 6 häufigsten IIPs gezählt. In diese Gruppe fallen weiterhin die idiopathische pulmonale Fibrose (IPF), die nichtspezifische interstitielle Pneumonie (NSIP), die respiratorische Bronchiolitis mit interstitieller Lungenerkrankung (RBILD), die desquamative interstitielle Pneumonie (DIP) und die akute interstitielle Pneumonie (AIP) [26].

Abzugrenzen hiervon sind umschriebene einzelne oder mehrere Foci einer organisierenden Pneumonie, welche zwar nicht auf eine bestimmte Ursache oder klinische Konstellation im Sinne einer sekundären organisierenden Pneumonie zurückgeführt werden können, sich aber aufgrund ihrer herdförmigen obliterans“ [19]. Der Begriff COP wurde 1983 von Davison et al. geprägt, wel- 

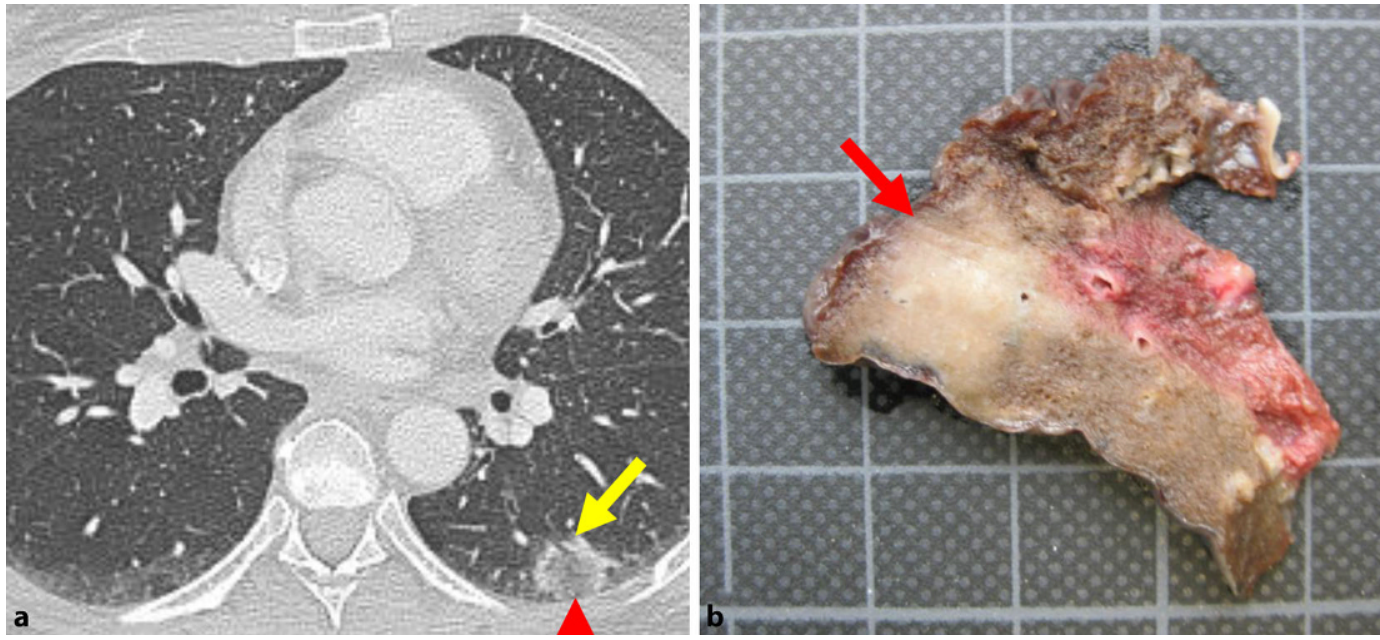

Abb. $1<$ Herd einer organisierenden Pneumonie (OP). a CT-Thorax in axialer Schichtung: OP mit AtollZeichen im linken Unterlappen mit peripherer randbildender Konsolidierung ( $g$ elber Pfeil), welche ein zentrales Milchglasinfiltrat begrenzt (rote Pfeilspitze). b Lungenteilresektat mit einem etwas unscharf begrenzten subpleuralen OPHerd (roter Pfeil)
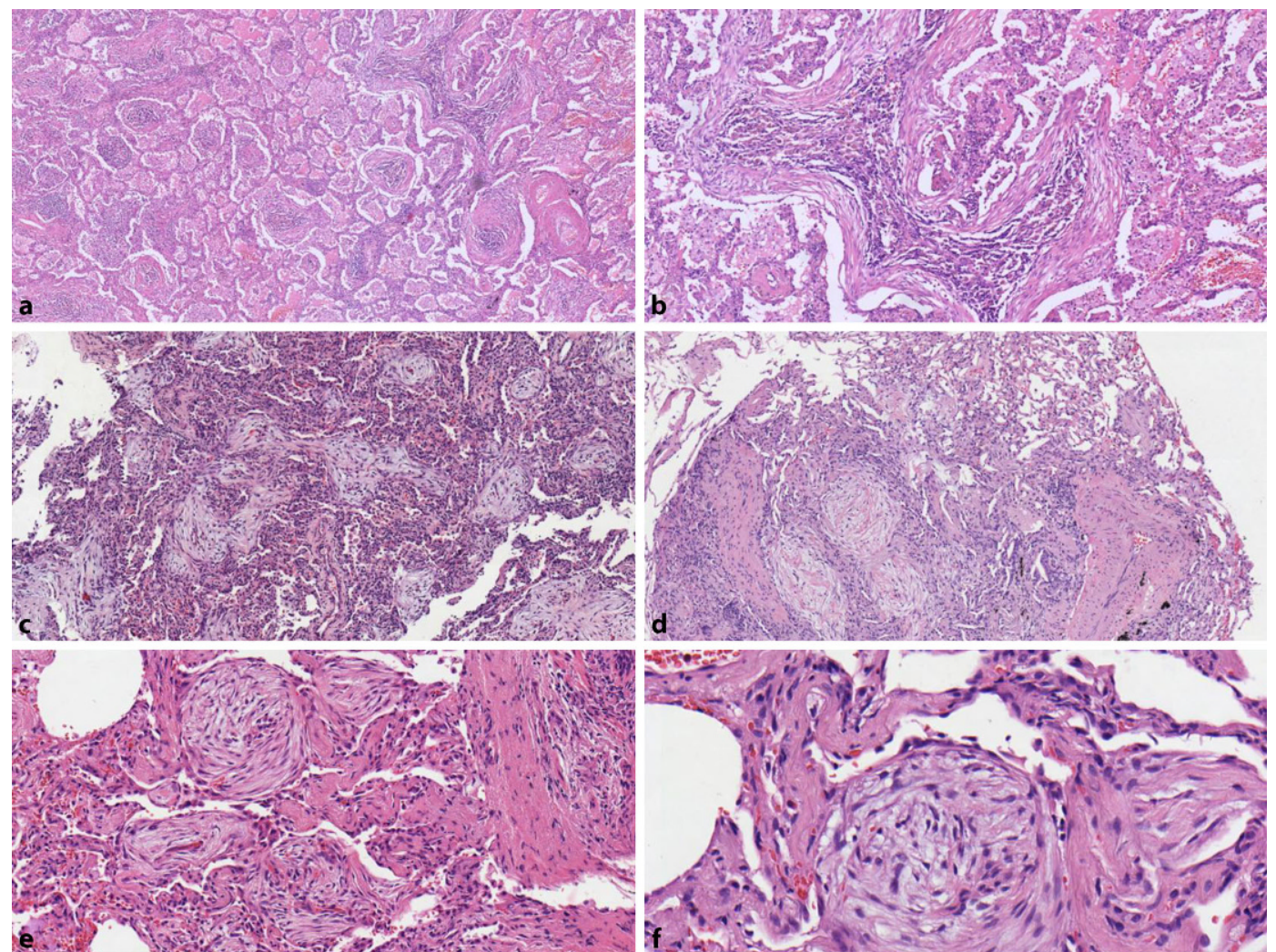

Abb. $2<$ Diagnostische Merkmale der organisierenden Pneumonie (OP). a, $b$ Erhaltene Lungenparenchymstruktur mit Mesenchymknospen und makrophagenreichem Begleitinfiltrat. c Zopfartige Verzweigung der Mesenchymknospen. d Fleckförmiges Verteilungsmuster. e Intraalveoläre Lokalisation der Mesenchymknospen mit sekundärer Pneu-

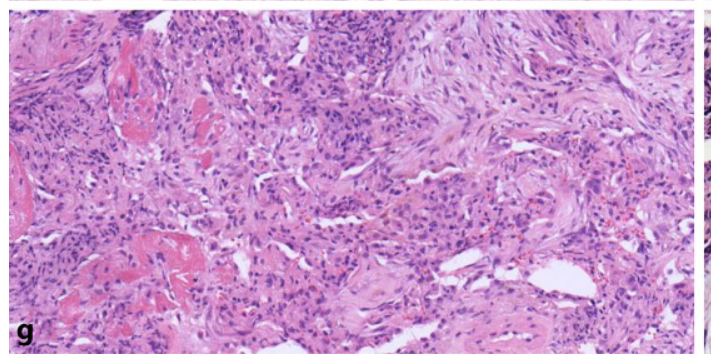

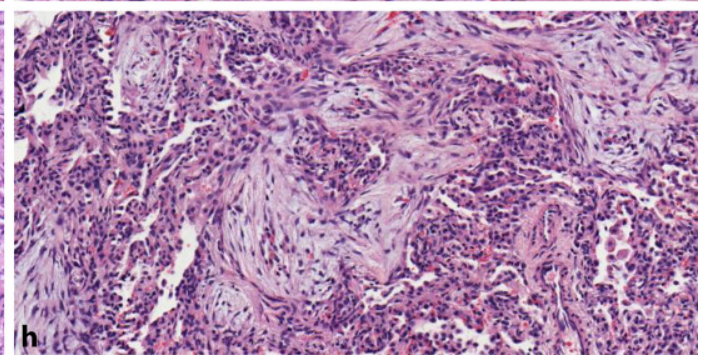
mozytenüberkleidung $(f)$. g Frühes Stadium der OP mit residuellen Fibrinpräzipitaten. $\mathbf{h}$ Mesenchymknospen mit Granulationsgewebe mit Kapillargefäßen, geringer chronischer Entzündung und mäßigem chronischen, interstitiellen Begleitinfiltrat 
Pathologe 2021 · 42:55-63 https://doi.org/10.1007/s00292-020-00903-8

(c) Springer Medizin Verlag GmbH, ein Teil von Springer Nature 2021

\section{R. Krupar · C. Kümpers · A. Haenel · S. Perner · F. Stellmacher}

\section{Kryptogen organisierende Pneumonie versus sekundäre organisierende Pneumonie}

\section{Zusammenfassung}

Die organisierende Pneumonie (OP) beschreibt ein histologisches Muster des akuten bzw. subakuten Lungenschadens. Klinisch fallen die Patienten mit Husten, Fieber und Dyspnoe auf. Es wird zwischen der idiopathischen bzw. kryptogen organisierenden (COP) und der sekundären organisierenden Pneumonie (OP) unterschieden. Bei der COP kann weder klinisch/radiologisch noch histologisch eine Ursache bestimmt werden. Sie wird zu den interstitiellen idiopathischen Pneumonien (IIP) gezählt nach Kriterien der American Thoracic Society (ATS) und der European Respiratory Society (ERS). Bei der sekundären organisierenden Pneumonie liegt ein bekannter auslösender Mechanismus vor, wie z. B. infektiöse Erreger, bestimmte Me- dikamente oder auch Begleiterscheinungen anderer primär pulmonaler Erkrankungen und Erkrankungen anderer Organsysteme. Beiden Formen gemeinsam ist das histologische Bild der intraalveolären Mesenchymknospen. Diese sind Myofibroblastenproliferate, welche sich zopfartig entlang der Alveolarräume verzweigen. Sie werden in der Regel von einem bis mäßigen interstitiellen und alveolären, chronischen und makrophagenreichen Entzündungszellinfiltrat begleitet. Wichtigste Differenzialdiagnose ist die gewöhnliche interstitielle Pneumonie (UIP). Diese zeigt ebenfalls Fibroblastenproliferate, welche allerdings interstitiell liegen. Die korrekte Zuordnung einer IIP als COP mittels klinischer, radiologischer und histologischer
Befunderhebung ist essenziell, da die COP im Gegensatz zur UIP sehr gut auf Kortikosteroide anspricht und somit gegenüber der UIP eine exzellente Prognose hat. Der Verlauf sekundärer organisierender Pneumonien hängt von der jeweiligen Grunderkrankung ab. Hier ist es wichtig, dass der Pathologe histologische Begleitcharakteristika einer OP korrekt identifiziert, um Hinweise auf eine mögliche Ursache geben zu können.

\section{Schlüsselwörter}

Idiopathische organisierende Pneumonie . Masson-Körper · Mesenchymknospen . Idiopathische interstitielle Pneumonie . Bronchiolitis obliterans mit organisierender Pneumonie (BOOP)

\section{Cryptogenic organizing pneumonia versus secondary organizing pneumonia}

\section{Abstract}

Organizing pneumonia (OP) describes a histological pattern of acute or subacute lung damage. Clinically, patients present with cough, fever, and dyspnea. A distinction is made between idiopathic or cryptogenic organizing pneumonia (COP) and secondary organizing pneumonia (OP). In COP, neither clinical/radiological nor histological causes can be determined. It is classified as an interstitial idiopathic pneumonia (IIP) according to the criteria of the American Thoracic Society (ATS) and the European Respiratory Society (ERS). Secondary organizing pneumonia has a known triggering mechanism, such as infectious agents, certain medications, or concomitant symptoms of other primary pulmonary diseases and diseases of other organ systems. Common to both forms is the histological picture of intra-alveolar mesenchymal buds. These are myofibroblast proliferates that branch out along the alveolar spaces. They are usually accompanied by a moderate interstitial and alveolar, chronic, and macrophage-rich inflammatory cell infiltrate. The most important differential diagnosis is common interstitial pneumonia (UIP). It also shows fibroblast proliferates, which are, however, located in the interstitium. The correct classification of an IIP as a COP by means of clinical, radiological, and histological findings is essential, since the COP, in contrast to the UIP, responds very well to corticosteroids and therefore has an excellent prognosis compared to the UIP. The course of secondary organizing pneumonia depends on the respective underlying disease. Here it is important for the pathologist to correctly identify potential accompanying histological characteristics in order to be able to provide clues to a possible cause of OP.

Keywords Idiopathic organizing pneumonia - Masson bodies - Fibroblast proliferation · Idiopathic interstitial pneumonia · Bronchiolitis obliterans organizing pneumonia (BOOP)
Begrenzung auch nicht für die Diagnose einer IIP qualifizieren (vgl. • Abb. 1).

\section{Klinik}

Beide Geschlechter sind in gleicher Häufigkeit von einer COP betroffen. Allerdings ist der Anteil an Nichtrauchern 2-mal häufiger im Vergleich zu Rauchern. Das mittlere Erkrankungsalter liegt bei 55 Jahren. In der Regel zeigen die Patienten subakute Symptome von Husten und Dyspnoe über eine Dauer von ca. 3 Monaten. Ein Teil der Patienten beschreibt zusätzlich vorausgegangene grippeähnliche Symptome wie Fieber, Halsschmerzen oder Schwäche. Weitere unspezifische Symptome sind Brustschmerzen, Nachtschweiß und milde Arthralgien. Ein Lungenfunktionstest zeigt milde bis mäßige restriktive Ventilationseinschränkungen $[4,14,17]$. In der bronchoalveolären Lavage (BAL) findet sich unter anderem eine erhöhte Gesamtzellzahl mit einer Lymphozytose und einer vermehrten Anzahl an Granulozyten. Zusätzlich lassen sich schaumzellige Makrophagen nachwei- sen. Die CD4/CD8-Ratio ist reduziert (mindestens $<0,9$ ). Insgesamt ähnelt das Bild der BAL bei einer Hypersensitivitätspneumonie [24].

\section{Radiologie}

In der Computertomographie (CT) ist die OP mit zahlreichen unterschiedlichen Bildmustern assoziiert. Die Verteilung der Befunde ist meist diffus und bilateral. Es treten jedoch auch fokale oder einseitige Anomalien auf [18]. $\mathrm{Zu}$ den häufigsten CT-Merkmalen gehören un- 

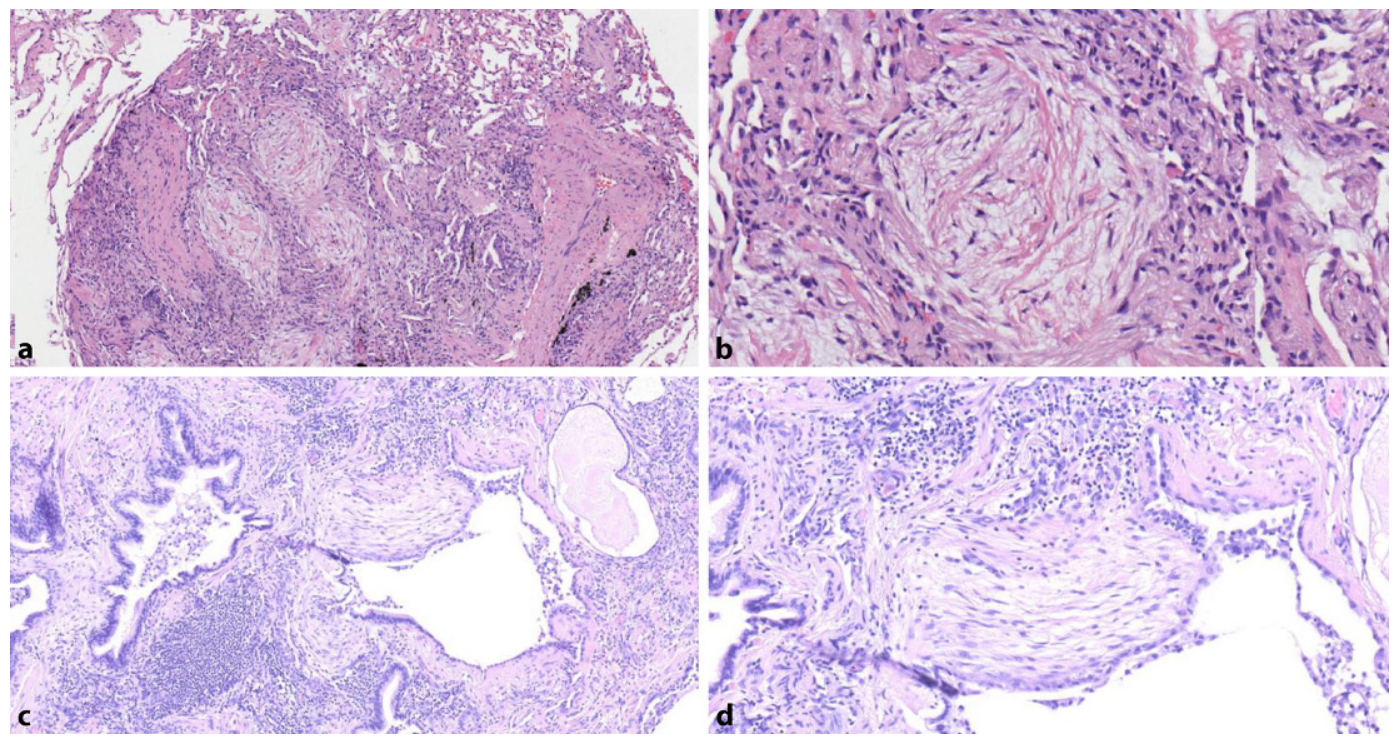

Abb. $3<$ Histologische Unterschiede der Mesenchymknospen der organisierenden Pneumonie $(\mathbf{a}, \mathbf{b})$ und der Fibroblastenfoci der gewöhnlichen interstitiellen Pneumonie (c, d) scharf berandete fleckige Konsolidierung mit einer vorwiegend subpleuralen oder peribronchialen Verteilung. Die Unterlappen sind in der Regel stärker betroffen. Seltener finden sich zentrilobuläre Mikronoduli und Rundherde [10]. Als hochspezifischer Befund gilt das AtollZeichen (umgekehrtes Halozeichen, siehe Abb. 1a), wenngleich die Sensitivität gering ist und es nur bei ca. $20 \%$ der Patienten mit einer OP auftritt [16].

Als CT-morphologische Differenzialdiagnosen sind die chronische eosinophile Pneumonie, das invasive Adenokarzinom, pulmonale Lymphome sowie die Sarkoidose zu nennen [10].

\section{Histologie}

Histologisches Leitmerkmal der OP sind Myofibroblastenproliferate (Masson-Körper) in Form zopfartiger Mesenchymknospen, welche die Alveolen zum Teil vollständig ausfüllen (vgl.

- Abb. 2). Hierbei handelt es sich um Granulationsgewebe aus spindelzelligem, fibromyxoidem Bindegewebe. Die Mesenchymknospen folgen dem Verlauf der Alveolarlichtungen und der respiratorischen Bronchioli. Sie bilden rundliche und ovale Zapfen in den Alveolen und können sich entlang der Alveolen in gewundenen Strukturen verzweigen. Schon in der Übersicht lassen sich diese Myofibroblastenproliferate gut erkennen, da die natürliche Struktur der Alveolarräume erhalten bleibt $[22,23]$.
Die intraalveoläre Lokalisation ist auch das wichtigste Unterscheidungsmerkmal der Mesenchymknospen der OP gegenüber den Fibroblastenfoci der UIP (vgl. Differenzialdiagnosen). Bislang ist die Pathophysiologie der Mesenchymknospen nicht endgültig geklärt. Der OP und der UIP gemeinsam ist eine mehr oder weniger defekte Wundheilung. Eine Verletzung von Alveolarepithelien führt zum Austritt von Fibrin und zum Einwandern von Entzündungszellen. Fibroblasteneinsprossungen aus den Alveolarsepten bilden im nächsten Schritt ein umgebendes Fasernetzwerk. Im weiteren Verlauf der Organisation entwickelt sich Granulationsgewebe mit Myofibroblasten sowie z.T. auch Kapillargefäßen zu dem charakteristischen Erscheinungsmuster der Mesenchymknospen [3]. Je nach Stadium ergeben sich somit unterschiedliche histologische Bilder mit einem variablen Entzündungszellinfiltrat aus Lymphozyten, Plasmazellen und Makrophagen innerhalb dieser Knospen. Zu einem frühen Zeitpunkt der Erkrankung können zusätzlich chronisch-entzündlich durchsetzte Fibrinexsudate in den Alveolen zu finden sein, welche von den hyalinen Membranen der DAD abgegrenzt werden müssen (vgl. Differenzialdiagnosen), oder auch schaumzellige Makrophagen [15]. Als eine Art Zwischenform von DAD und OP ist die akute fibrinöse und organisierende Pneumonie (AFOP) zu erwähnen. Hier finden sich neben intraalveolären Fibrinansammlungen (sogenannte Fibrinbälle) zusätzlich lockere Fibroblastenproliferate der OP in einem fleckförmigen Verteilungsmuster [20].

Die Veränderungen der OP sind entlang der respiratorischen Bronchiolen und peribronchial lokalisiert und werden zusätzlich von einem variablen, meist nur schwachen bis mäßigen interstitiellen Entzündungszellinfiltrat aus Lymphozyten und Plasmazellen begleitet. Typischerweise ist die Lunge fleckförmig befallen mit dazwischen gelegenen Arealen von regelhaftem Lungenparenchym. Selten kann auch ein diffuser, flächiger Befall der Lunge vorliegen [23].

Es ist wichtig zu betonen, dass das histologische Bild der OP lediglich ein spezifisches Muster beschreibt. Die Differenzierung einer COP von einer sekundären OP erfolgt dann durch den Nachweis zusätzlicher histologischer Charakteristika (vgl. sekundäre OP), wie zum Beispiel den Nachweis von Granulomen und mehrkernigen Riesenzellen als Zeichen einer Hypersensitivitätspneumonie. Alternativ müssen für die finale Diagnose einer COP alternative Ursachen eines OP-Musters unter Kenntnis der pathologischen, radiologischen und klinischen Befunde ausgeschlossen werden. Dementsprechend sollte im pathologischen Befundbericht bei ausschließlichem Vorliegen eines OPMusters und ohne Kenntnis weiterer klinischer, anamnestischer oder bildgebender Informationen zur Genese 
Tab. 1 Histologische Unterscheidungsmerkmale von Mesenchymknospen der organisierenden Pneumonie (OP) und Fibroblastenfoci der gewöhnlichen interstitiellen Pneumonie (UIP)

\section{OP}

Veränderungsmuster

Fleckförmige Verteilung

Zeitliche Heterogenität

Ja

Fibroblastenproliferate

Verteilung

Flächig

Lokalisation

Intraalveolär

Form

Entzündung
UIP

Ja

Ja

Interstitiell

Kuppelartig

Unterschiedlich, eher wenig
Einzeln dann der Terminus OP/COP verwendet werden [26].

\section{Differenzialdiagnosen}

Die UIP ist wahrscheinlich die wichtigste Differenzialdiagnose der OP aufgrund ihres deutlichen Unterschieds hinsichtlich Therapie und Prognose. In Lungenteilresektaten und größeren Biopsien solle die Differenzierung keine Schwierigkeiten bereiten. Durch das zwar fleckförmige, aber in sich homogene Bild der OP ohne eine zeitliche Heterogenität und mit erhaltener Grundstruktur des Lungenparenchyms unterscheidet sie sich in der Regel schon in der Übersicht deutlich von einer UIP. Schwieriger ist die Interpretation bei kleinen bzw. gequetschten Biopsien, in denen nur einzelne Merkmale der beiden IIPs repräsentiert sind. OP und UIP sind beide gekennzeichnet durch Fibroblastenproliferate mit einem bisweilen sehr ähnlichen histologischen Erscheinungsbild (vgl. - Abb. 3). Unterschiedlich ist jedoch ihre Lokalisation. Die Mesenchymknospen der OP liegen in den Alveolen und respiratorischen Bronchioli und zeigen nur abschnittsweise eine Verbindung zu benachbarten Alveolarsepten. Im Gegensatz hierzu befinden sich die Fibroblastenfoci der UIP im Interstitium und sind dementsprechend durch Typ-2-Pneumozyten überkleidet. Leider hat sich dies nicht als diagnostisches Unterscheidungskriterium einer OP von einer UIP bewährt, da auch Mesenchymknospen der OP häufig im Verlauf eine sekundäre Überkleidung durch Typ-2-Pneumozyten zeigen (vgl. Abb. 2f). Somit ist auch eine CK7oder TTF1-Färbung zur Unterscheidung von Fibroblastenfoci und Mesenchym- knospen nicht hilfreich. $\bullet$ Tab. 1 gibt eine Übersicht der Charakteristika, welche auch im Falle von kleinen Biopsien bei der korrekten Unterscheidung von Mesenchymknospen der OP und Fibroblastenfoci der UIP unterstützen können. Wichtig hierbei ist zu betonen, dass es zwar legitim ist, eine OP auch an kleinen Lungengewebefragmenten zu diagnostizieren, die Diagnose eines UIP-Musters sollte im Gegensatz hierzu jedoch ausschließlich an größeren Gewebeproben wie Kryobiopsaten oder Lungenteilresektaten, erfolgen.

Eine weitere differenzialdiagnostische Schwierigkeit kann der DAD in organisierender Phase darstellen. $\mathrm{Zu}$ diesem Zeitpunkt liegen die für den DAD typischen hyalinen Membranen allenfalls nur noch vereinzelt vor. Dagegen sind in den Alveolarsepten Fibroblastenproliferate zu finden, welche zu Verwechslungen mit den Mesenchymknospen der OP führen können. Histologisch hilft hier der fokale Nachweis von hyalinen Membranen weiter, welche für den DAD diagnostisch sind. Ferner sind die Veränderungen des DAD im Stadium der Organisation durch meist längliche, in den Septen verzweigte Fibroblastenproliferate charakterisiert, die in aller Regel nicht bis in die Alveolarräume hineinreichen. Weiteren Aufschluss geben klinische und radiologische Informationen, wie ein Z.n. Beatmung oder eine diffuse Veränderung beider Lungen in der Bildgebung [22].

Eine differenzialdiagnostische Herausforderung kann das NSIP (nichtspezifische interstitielle Pneumonie)/OPÜberlappungsmuster darstellen. Hier findet sich histologisch, neben intraalveolären Mesenchymknospen der $\mathrm{OP}$, eine räumlich und zeitlich homogene alveolarseptale Fibrose mit interstitieller Entzündung entsprechend eines NSIPMusters [11]. Hilfreich für die Abgrenzung einer OP von einem NSIP/OPÜberlappungsmuster ist dann die radiologische Bildgebung. Während Konsolidierungen eher für eine $\mathrm{OP}$ sprechen, sind Milchglastrübungen, Retikulationen und Traktionsbronchiektasen in differenzialdiagnostisch schwierigen Fällen hinweisende Veränderungen für ein NSIP/OP-Überlappungsmuster [25].

\section{Therapie und Prognose}

Die COP ist glukokortikoidsensitiv und hat eine sehr gute Prognose. Therapeutisch werden orale oder auch venöse Kortikosteroide mit konstanter Initialdosis für 4-8 Wochen eingesetzt, gefolgt von einem langsamen Ausschleichen über 3-12 Monate. COP-Patienten sollten ein schnelles Ansprechen (innerhalb von Tagen) auf die Therapie zeigen. Rezidive sind häufig (13-58\%) und entstehen in der Regel bei Beendigung der Therapie. Sie haben allerdings keinen Einfluss auf die exzellente Prognose der COP. Aber sie legen ein enges klinisches und radiologisches Monitoring der Patienten während der Therapiephase nahe. Zusätzlich sollten ein Rezidiv oder ein fehlendes Ansprechen auf Glukokortikoide Anlass geben, die Diagnose einer $\mathrm{COP}$ noch einmal genau zu überprüfen und andere Ursachen einer OP sicher auszuschließen. Die fokale COP in Form eines umschriebenen Lungenrundherds bedarf keiner weiteren Therapie nach Exzision [3, 6].

\section{Sekundäre OP}

Sekundäre OP umfassen eine heterogene Gruppe unterschiedlicher infektiöser und nichtinfektiöser Erkrankungen, deren Gemeinsamkeit das radiologische und histologische OP-Muster ist. In vergleichenden Studien sind COPs in der Regel etwas häufiger vertreten als sekundäre OPs (ca. $2 / 3$ zu 1/3) $[2,9,21]$. Signifikante Unterschiede hinsichtlich Patientencharakteristika sowie klinischen Symptomen und ihre Schweregrade finden sich jedoch nicht zwischen den beiden Formen. Auch die radio- 


\section{Schwerpunkt: Nicht-neoplastische Lungenerkrankungen}

Tab. 2 Sekundäre organisierenden Pneumonien und Sonderformen der kryptogen organisierenden Pneumonie

\begin{tabular}{l} 
Ursachen \\
\hline Infektiös \\
Bakterien \\
Viren \\
Parasiten \\
Pilze \\
Exogene Noxen \\
Medikamente \\
\hline $\begin{array}{l}\text { Inhalative Noxen } \\
\text { Bestrahlung }\end{array}$ \\
\hline $\begin{array}{l}\text { Systemische Erkran- } \\
\text { kungen und Begleit- } \\
\text { reationen bei ande- } \\
\text { gen } \\
\text { Kollaganerkrankun- }\end{array}$ \\
\hline
\end{tabular}

Kollagenosen

Details

Erreger

Streptococcus pneumoniae, atypische Mykobakterien (Mycobacterium avium), Actinomyces israelii, Chlamydia pneumoniae, Coxiella burnetii, Legionella pneumophila, Mycoplasma pneumonia, Nocardia asteroides, Staphylococcus aureus, Serratia marcescens, Pseudomonas aeruginosa

HIV, Influenza, Parainfluenza, Herpes, HCV, SARS-CoV-2

Plasmodium vivax

Cryptococcus neoformans, Penicillium janthinellum, Pneumocystis jiroveci (bei AIDS)

\section{Auslöser}

www.pneumotox.com, aktuell mit 114 Kausalmedikamenten, z. B.: Amiodaron, Nitrofurantoin, Bleomycin, Chemotherapeutika, Immuncheckpoint-Inhibitoren

Kokain, E-Zigaretten, NO, Ammoniak

v. a. 3-6 Monate nach Brustbestrahlung in 2,5\% der Mammakarzinompatienten mit Radiatio

\section{Grunderkrankung}

CED u. ähnl.

Transplantation

Hämatologische Neoplasien

Begleiterscheinung bei anderen pulmonalen Erkrankungen

Malignom, Abszess, Infarkt, Pilzinfektion (z. B. Aspergillom)

Granulomatose mit Polyangiitis (GPA, Wegener-Granulomatose)

Aspirationspneumonie

v. a. rheumatoide Arthritis, Dermatomyositis/Polymyositis, aber auch SLE, CREST-, Sjögren-Syndrom

$U C, M C, P B C$

Lunge, Leber, Knochenmark

\section{Z. B. bei Hodgkin und Non-Hodgkin-Lymphomen, AML, MDS}

Randbereich eines Herdes mit umgebender OP als Begleitreaktion

2 Formen:

GPA mit Nekrosen und Aspekten einer OP

BOOP-artige Granulomatose mit minimalen bzw. keinen Nekrosen

Häufige Ursache einer OP, klinisch oft nicht bekannt

Hypersensitivitätspneumonitis
OP mit:

Chronischer interstitieller Entzündung

Nichtnekrotisierenden Epitheloidzellgranulomen

Mehrkernige Riesenzellen
Anmerkungen

$-$

Häufige Ursache eines OP-Musters bei länger zurückliegender, nicht mehr aktiver Infektion mit inkompletter Remission

Korrelation zu Anamnese und laborchemischen Parametern (Antikörpertiter)

Korrelation zu klinischen und laborchemischen Parametern

Pilznachweis mit Sonderfärbungen (PAS, Versilberung), Korrelation Mikrobiologie oder Erregernachweis am Paraffinmaterial durch PCR

Klinische Korrelation notwendig $\rightarrow$ Besserung nach Absetzen?

Bei Chemotherapeutika und ImmuncheckpointInhibitoren Ausschluss Malignität bzw. Tumorprogress

Klinische Korrelation

Kann außerhalb des Bestrahlungsfensters liegen und ist GK-sensitiv $\leftrightarrow$ Abgrenzung zur Strahlenpneumonitis

Klinische Korrelation

Klinische Korrelation, andere Organbefunde Z.B. bei akuter Abstoßung n. Lungentransplantation

V.a. nach Chemotherapie und Knochenmarkstransplantation

Achtung vor falsch benignen Ergebnissen bei V.a. $\mathrm{BC} \rightarrow$ Korrelation mit Radiologie und ggf. erneute Probenentnahme

Zusätzlich Fibrose mit chronischer, granulomatöser Entzündung, Riesenzellen und nekrotisierender Vaskulitis

Peribronchiales Verteilungsmuster, Fremdkörpergranulome mit mehrkernigen Riesenzellen (polarisationsoptischer Nachweis von z. B. Nahrungsbestandteilen) und Neutrophile

Erkennen der histologischen Merkmale als wichtiger Beitrag zur korrekten Einordnung 


\begin{tabular}{|c|c|c|}
\hline Ursachen & Details & Anmerkungen \\
\hline IIP mit OP & Histologie & - \\
\hline UIP & $\begin{array}{l}\text { DAD- oder OP-Muster bei akuter Exazerbation einer bekannten UIP oder } \\
\text { bei Erstdiagnose mit akutem Lungenversagen }\end{array}$ & $\begin{array}{l}\text { Suche nach präexistentem UIP-Muster (Fibrose, } \\
\text { Honigwabenmuster) }\end{array}$ \\
\hline Sonderformen der OP & Histologie & - \\
\hline \multirow[t]{2}{*}{$\begin{array}{l}\text { Akute fibrinöse und OP } \\
\text { (AFOP) }\end{array}$} & \multirow[t]{2}{*}{$\begin{array}{l}\text { OP mit intraalveolären Fibrinpropfen mit fleckförmiger Verteilung und } \\
\text { Typ-2-Pneumozyten-Hyperplasie }\end{array}$} & $\begin{array}{l}\text { Ähnliche Klinik wie DAD, aber histologisch keine } \\
\text { hyalinen Membranen }\end{array}$ \\
\hline & & Langzeitveränderung bei SARS-CoV-2 \\
\hline Fibrosierende COP & Seltene Erkrankung mit OP und interstitieller Fibrose & Progredientes Atemversagen, reagiert nicht auf GK \\
\hline Akute fulminante COP & OP-Muster ohne Fibrose & $\begin{array}{l}\text { Klinisch wie ARDS mit akutem Atemwegsversagen, } \\
\text { in der Regel GK-sensitiv }\end{array}$ \\
\hline $\begin{array}{l}\text { Chronische eosinophile } \\
\text { Pneumonie mit OP }\end{array}$ & $\begin{array}{l}\text { Überschneidungssyndrom von chronischer eosinophiler Pneumonie } \\
\text { und OP mit intraalveolärer Ansammlung von Eosinophilen und OP- } \\
\text { Muster }\end{array}$ & $\begin{array}{l}\text { Ursachen: idiopathisch, infektiös, medikamentös, } \\
\text { Immunreaktion, GK-sensitiv }\end{array}$ \\
\hline \multicolumn{3}{|c|}{$\begin{array}{l}\text { AML akute myeloische Leukämie, } A R D S \text { „acute respiratory distress syndrome“, BC Bronchialkarzinom, BOOP Bronchiolitis obliterans mit organisierender Pneu- } \\
\text { monie, CED chronisch entzündliche Darmerkrankung, COP kryptogen organisierende Pneumonie, DAD diffuser Alveolarschaden, IIP interstitielle idiopathische } \\
\text { Pneumonie, GK Glukokortikoide, MC Morbus Crohn, MDS myelodysplastisches Syndrom, OP organisierende Pneumonie, PBC primäre biliäre Zirrhose, SLE sys- } \\
\text { temischer Lupus erythematodes, UC Colitis ulcerosa, UIP gewöhnliche interstitielle Pneumonie }\end{array}$} \\
\hline
\end{tabular}

logische Bildgebung kann häufig nicht weiter zwischen den beiden Gruppen differenzieren, wobei Baha et al. in ihrer Studie angeben, dass Lungenveränderungen in Patienten mit sekundärer OP häufiger in zentralen und mittleren Lungenabschnitten zu finden sind [2]. Drakopanagiotakis et al. fanden in ihrer vergleichenden Analyse neben Unterschieden in der Blutserumanalyse (z.B. höherer Kalium-, Natriumgehalt, höhere Thrombozytenkonzentration etc.) in der BAL von Patienten mit einer sekundären OP häufiger eine Lymphozytose [9]. Diesbezüglich sind aber sicherlich weder radiologische noch laborchemische Parameter allein geeignet zwischen den beiden Formen der OP zu unterscheiden. COPs und sekundäre OPs sind in der Regel beide glukokortikoidsensitiv, wobei nach einer Studie von Lohr et al. Therapieansprechen und Prognose bei COPs besser ist [21]. Hier stellt bei sekundären OPs sicherlich die jeweils assoziierte Grunderkrankung den entscheidenden Faktor dar bezüglich Therapieverhalten und Langzeitüberleben. • Tab. 2 enthält eine Zusammenfassung der wichtigsten Ursachen einer sekundären $\mathrm{OP}$, deren histologische Charakteristika und relevante Unterschiede hinsichtlich Therapie und Prognose. Neben erregerbedingten Ursachen (diverse Bakterien, Pilze, Viren) spielen exogene Noxen wie zahlreiche Medikamente oder ionisierende Strahlung eine Rolle. Eine Reihe pri- märer pulmonaler Erkrankungen (z.B. Aspirationspneumonien, Tumore, Granulomatose mit Polyangiitis/WegenerGranulomatose) können ein OP-Muster zeigen. Zusätzlich kann sich ein OPMuster bei anderen IIPs finden, wie bei einer akuten Exazerbation einer UIP, was bei kleinen Biopsien schwer von einem NSIP-Muster zu unterscheiden sein kann. Zuletzt gibt es zahlreiche primär nicht pulmonale Krankheiten, z.B. hämatologische Neoplasien oder Erkrankungen aus dem rheumatischen Formenkreis, welche sich unter anderem auch durch eine OP manifestieren können [7]. Wichtig zu erwähnen sind hier insbesondere systemische Bindegewebserkrankungen (Kollagenosen), die bei pulmonaler Beteiligung neben einem OP-Muster unter anderem auch ein NSIP/OP-Überlappungsmuster zeigen können im Rahmen einer interstitiellen Pneumonie mit autoimmunen Eigenschaften („interstitial pneumonia with autoimmune features“, IPAF) [12]. In der Zusammenschau kann die Histologie nur in einem Teil der Fälle bei der korrekten Entitätszuordnung helfen (•Abb. 4). Wichtige Hilfestellung für den betreuenden Kliniker kann der Pathologe zum Beispiel durch das Erkennen einer Hypersensitivitätspneumonie oder einer Aspirationspneumonie geben. In der Regel ist für die abschließende Einordnung einer sekundären OP die enge Korrelation mit den klinischen und anamnestischen Befunden sowie der Bildgebung notwendig. Dies ist relevant, da sich unterschiedliche Formen der sekundären OP deutlich hinsichtlich ihrer Therapiestrategie und ihrer Prognose unterscheiden.

\section{Fazit für die Praxis}

- Die organisierende Pneumonie (OP) ist ein histologisches Muster, welches idiopathisch als kryptogen organisierende Pneumonie (COP) auftreten kann oder sekundär im Rahmen einer Reihe von pulmonalen und nichtpulmonalen Grunderkrankungen.

- Die COP wird zu den idiopathischen interstitiellen Pneumonien (IIP) nach ATS/ERS-Leitlinien gezählt.

- Das histologische Hauptmerkmal der OP sind intraalveoläre Mesenchymknospen.

- Wichtigste Differenzialdiagnose ist die gewöhnliche interstitielle Pneumonie (UIP) mit intraseptalen Fibroblastenfoci.

- Die COP ist glukokortikoidsensitiv und hat eine exzellente Prognose.

- Die Histologie kann bei der korrekten Zuordnung einer sekundären OP eine wichtige Rolle spielen. 


\section{Schwerpunkt: Nicht-neoplastische Lungenerkrankungen}
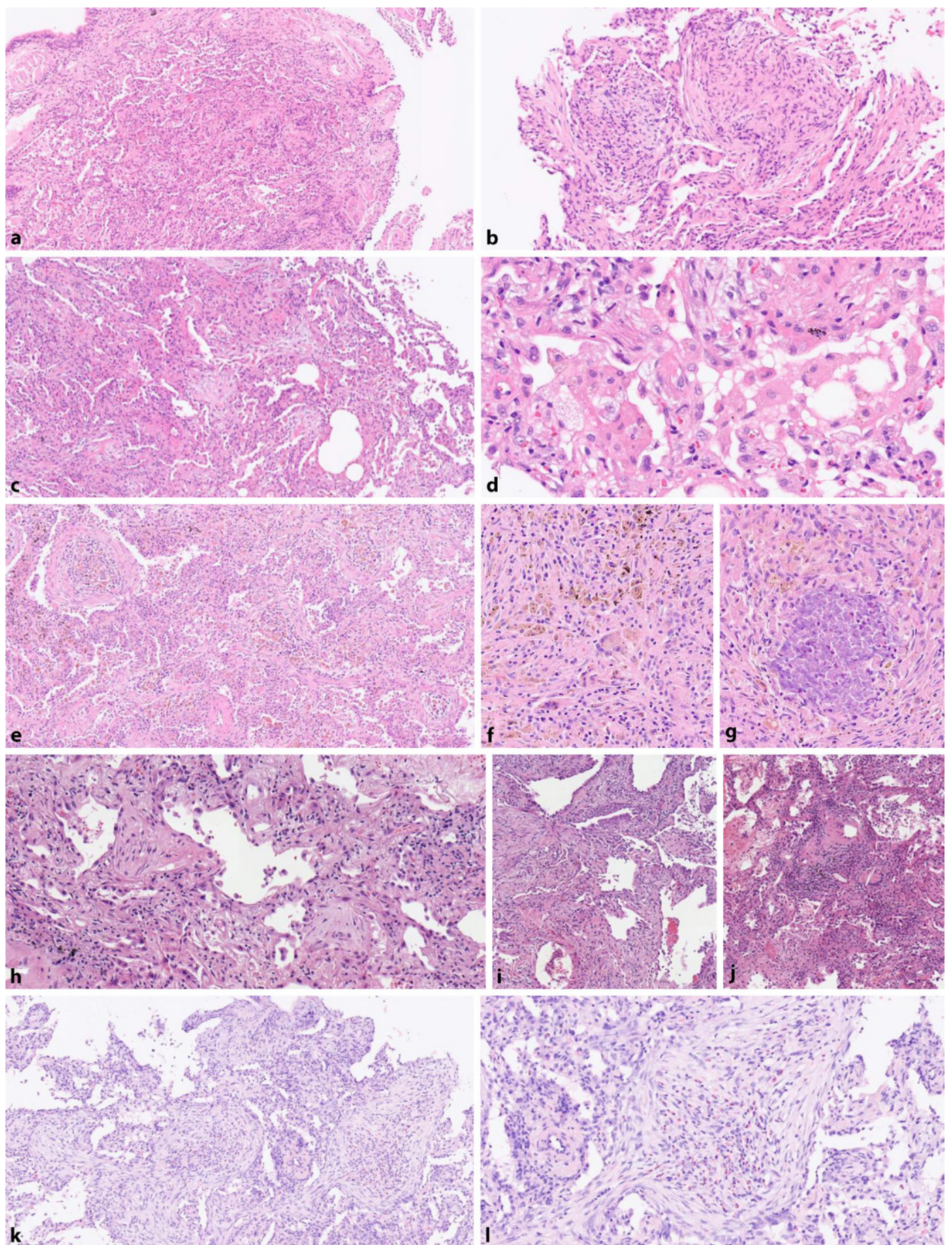

Abb. $4<$ Sekundäre organisierende Pneumonie (OP). a OP bei Mycobacterium-aviumintracellulare(MAI)-Infektion mit Nachweis nichtnekrotisiender Epitheloidzellgranulome (b) c OP unter AmiodaronTherapie mit schaumzelligen Makrophagen (d). e Aspirationspneumonie mit OP-Muster, Fremdkörperriesenzellen (f) und teils floridem Begleitinfiltrat (g). h, i Hypersensitivitätspneumonitis mit OP, Granulomen, mehrkernigen Riesenzellen mit Einschlüssen und chronischem Begleitinfiltrat (j). k, IChronische eosinophile Pneumonie mit OP 


\section{Korrespondenzadresse}

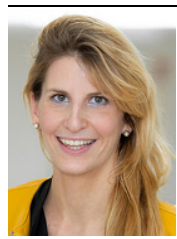

Rosemarie Krupar

Pathologie, Forschungszentrum Borstel, Leibniz Lungenzentrum

Parkallee 3A, 23845 Borstel,

Deutschland

rkrupar@fz-borstel.de

\section{Einhaltung ethischer Richtlinien}

Interessenkonflikt. R. Krupar, C. Kümpers, A. Haenel, S. Perner und F. Stellmacher geben an, dass kein Interessenkonflikt besteht.

Für diesen Beitrag wurden von den Autoren keine Studien an Menschen oder Tieren durchgeführt. Für die aufgeführten Studien gelten die jeweils dort angegebenen ethischen Richtlinien.

\section{Literatur}

1. American Thoracic Society, European Respiratory Society (2002) American thoracic society/ European respiratory society international multidisciplinary consensus classification of the idiopathic interstitial pneumonias. This joint statement of the American thoracic society (ATS), and the European respiratory society (ERS) was adopted by the ATS board of directors, June 2001 and by the ERS executive committee, June 2001. Am J Respir Crit Care Med 165:277-304. https:// doi.org/10.1164/ajrccm.165.2.ats01

2. Baha A, Yıldırım F, Köktürk N et al (2018) Cryptogenic and secondary organizing pneumonia: clinical presentation, radiological and laboratory findings, treatment, and prognosis in 56 cases. Turk Thorac 19:201-208. https://doi.org/10.5152/TurkThoracJ. 2018.18008

3. Chandra D, Maini R, Hershberger DM (2020) Cryptogenic organizing pneumonia. StatPearls Publishing, Treasure Island (FL)

4. Cordier JF, Loire R, Brune J (1989) Idiopathic bronchiolitis obliterans organizing pneumonia. Definition of characteristic clinical profiles in a series of 16 patients. Chest 96:999-1004. https:// doi.org/10.1378/chest.96.5.999

5. Costabel U, Guzman J (1991) BOOP: what is old, what is new? Eur Respir J 4:771-773

6. Cottin V, Cordier J-F (2012) Cryptogenic organizing pneumonia. Semin Respir Crit Care Med 33:462-475. https://doi.org/10.1055/s-0032 1325157

7. Daniels CE, Myers JL, Utz JP et al (2007) Organizing pneumonia in patients with hematologic malignancies: a steroid-responsive lesion. Respir Med 101:162-168. https://doi.org/10.1016/j. rmed.2006.03.035

8. Davison AG, Heard BE, McAllister WA, TurnerWarwick ME (1983) Cryptogenic organizing pneumonitis. Q JMed 52:382-394

9. Drakopanagiotakis F, Paschalaki K, Abu-Hijleh Met al (2011) Cryptogenic and secondary organizing pneumonia: clinical presentation, radiographic findings, treatment response, and prognosis. Chest 139:893-900. https://doi.org/10.1378/chest.100883
10. ElickerBM, WebbWR(2013) Fundamentals of highresolution lung $C T$ : common findings, common patterns, common diseases, and differential diagnosis, 1. Aufl. Lippincott Williams \& Wilkins, Philadelphia, PA

11. Enomoto N, Sumikawa $H$, Sugiura $H$ et al (2020) Clinical, radiological, and pathological evaluation of "NSIP with OP overlap" pattern compared with NSIP in patients with idiopathic interstitial pneumonias. Respir Med 174:106201. https://doi. org/10.1016/j.rmed.2020.106201

12. Fischer A, Antoniou KM, Brown KK et al (2015) An official European respiratory society/American thoracic society research statement: interstitial pneumonia with autoimmunefeatures. EurRespir J 46:976-987. https://doi.org/10.1183/13993003. 00150-2015

13. Gudmundsson G, Sveinsson O, Isaksson $\mathrm{HJ}$ et al (2006) Epidemiology of organising pneumonia in Iceland. Thorax 61:805-808. https://doi.org/10. 1136/thx.2006.059469

14. Izumi T, Kitaichi M, Nishimura K, Nagai S (1992) Bronchiolitis obliterans organizing pneumonia. Clinical features and differential diagnosis. Chest 102:715-719. https://doi.org/10.1378/chest.102. 3.715

15. Katzenstein A-LA (2016) Diagnostic atlas of nonneoplastic lung disease: a practical guide for surgical pathologists, 1. Aufl. DEMOS HEALTH, New York, NY

16. Kim SJ, Lee KS, Ryu YH et al (2003) Reversed halo sign on high-resolution CT of cryptogenic organizing pneumonia: diagnostic implications. AJR Am J Roentgenol 180:1251-1254. https://doi. org/10.2214/ajr.180.5.1801251

17. King TE, Mortenson RL (1992) Cryptogenic organizing pneumonitis. The North American experience. Chest 102:8S-13S

18. Kligerman SJ, Franks TJ, Galvin JR (2013) From the radiologic pathology archives: organization and fibrosis as a response to lung injury in diffuse alveolar damage, organizing pneumonia, and acute fibrinous and organizing pneumonia. Radiographics 33:1951-1975. https://doi.org/10. 1148/rg.337130057

19. Lange W (1901) Ueber eine eigenthumliche Erkrankung der kleinen Bronchien und Bronchiolen (Bronchitis et Bronchiolits Obliterans). Dtsch Arch Klin Med 70:342-364

20. Leslie KO, Wick MR (2011) Practical pulmonary pathology: a diagnostic approach, 2. Aufl. Elsevier, Saunders

21. Lohr RH, Boland BJ, Douglas WW et al (1997) Organizing pneumonia. Features and prognosis of cryptogenic, secondary, and focal variants. Arch Intern Med 157:1323-1329. https://doi.org/10. 1001/archinte.157.12.1323

22. Mukhopadhyay S (2016) Non-neoplastic pulmonary pathology with online resource, 1 . Aufl. Cambridge University Press,

23. Perner S, Stellmacher F, Jongik D, Rabe K-F (2018) Nicht-neoplastische/interstitielle Lungenerkrankungen. Internationale Akademie für Pathologie Deutsche Abteilung e.V., Herbsttutorial 2018:516

24. Poletti V, Cazzato S, Minicuci N et al (1996) The diagnostic value of bronchoalveolar lavage and transbronchial lung biopsy in cryptogenic organizing pneumonia. Eur Respir J 9:2513-2516. https://doi.org/10.1183/09031936.96.09122513

25. Todd NW, Marciniak ET, Sachdeva A et al (2015) Organizing pneumonia/non-specific interstitial pneumonia overlap is associated with unfavorable lung disease progression. Respir Med
109:1460-1468. https://doi.org/10.1016/j.rmed. 2015.09.015

26. Travis WD, Costabel U, Hansell DM et al (2013) An official American thoracic society/European respiratory society statement: update of the international multidisciplinary classification of the idiopathic interstitial pneumonias. Am J Respir Crit Care Med 188:733-748. https://doi.org/10.1164/ rccm.201308-1483ST 